\title{
PHOX2B is a suppressor of neuroblastoma metastasis
}

\author{
Osnat Naftali ${ }^{1}$, Shelly Maman ${ }^{1}$, Tsipi Meshel ${ }^{1}$, Orit Sagi-Assif ${ }^{1}$, Ravit Ginat ${ }^{1}$, Isaac \\ P. Witz ${ }^{1}$ \\ ${ }^{1}$ Department of Cell Research and Immunology, The George S. Wise Faculty of Life Sciences, Tel Aviv University, Tel Aviv, \\ Israel 69978 \\ Correspondence to: Isaac P. Witz, e-mail: isaacw@tavex.tau.ac.il \\ Keywords: $\mathrm{PHOX2B}$, minimal residual disease, metastasis, neuroblastoma, methylation \\ Received: December 17, $2015 \quad$ Accepted: January 23, $2016 \quad$ Published: January 28, 2016
}

\section{ABSTRACT}

Paired like homeobox 2B (PHOX2B) is a minimal residual disease (MRD) marker of neuroblastoma. The presence of MRD, also referred to as micro-metastases, is a powerful marker of poor prognosis in neuroblastoma.

Lung metastasis is considered a terminal event in neuroblastoma. Lung micrometastatic neuroblastoma (MicroNB) cells show high expression levels of PHOX2B and possess a less malignant and metastatic phenotype than lung macro metastatic neuroblastoma (MacroNB) cells, which hardly express PHOX2B.

In vitro assays showed that PHOX2B knockdown in MicroNB cells did not affect cell viability; however it decreased the migratory capacity of the MicroNBshPHOX2B cells. An orthotopic inoculation of MicroNB-shPHOX2B cells into the adrenal gland of nude mice resulted in significantly larger primary tumors and a heavier micro-metastatic load in the lungs and bone-marrow, than when control cells were inoculated.

PHOX2B expression was found to be regulated by methylation. The PHOX2B promoter in MacroNB cells is significantly more methylated than in MicroNB cells. Demethylation assays using 5-azacytidine demonstrated that methylation can indeed inhibit PHOX2B transcription in MacroNB cells.

These pre-clinical data strongly suggest that РНОХ2B functions as a suppressor of neuroblastoma progression.

\section{INTRODUCTION}

Neuroblastoma is the most common extracranial solid tumor in children, and accounts for approximately $15 \%$ of all childhood cancer deaths $[1,2]$. Most children diagnosed with neuroblastoma over the age of one year present metastatic disease, when the presence of lung metastases is considered a rare and terminal event $[3,4]$. Most patients with metastatic disease who achieve near complete remission typically relapse because of the presence of neuroblastoma micrometastasis, also known as minimal residual disease (MRD) [5-8]. Cure after clinical relapse is rare [9].

The current consensus is that micro-metastases remain in a dormant state, until "awakened" to progress towards overt metastases [10]. Understanding the mechanism regulating the progression of micro-metastases to overt metastasis is crucial for the development of efficient modalities to monitor and treat neuroblastoma metastasis.
The ideal MRD marker is one that is tumor specific and has no expression in the normal compartments. Stutterheim et al. (2008) found PHOX2B to be superior to TH and GD2 synthase, the commonly used MRD markers, in specificity and sensitivity of neuroblastoma MRD detection [11].

PHOX2B is a homeodomain transcription factor that promotes differentiation in neural crest cells [12]. PHOX2B was the first gene for which germline mutations - such as heterozygous missense and nonsense mutations - were found in patients with neuroblastoma $[13,14]$. Subtyping neuroblastoma tumors indicated that low expression of PHOX2B is associated with higher tumor stage, poor outcome and poor survival [15].

We previously described the development of a mouse model for human neuroblastoma metastasis. An orthotopic inoculation of the human neuroblastoma cell line MHH-NB-11 [16] to the adrenal gland of 
athymic nude mice yielded local adrenal tumors, as well as lung metastasis. After several cycles of in vivo passages of cells cultured from these local tumors and lung metastases, local and lung metastatic variants were generated [17]. Nude mice inoculated orthotopically with neuroblastoma lung metastatic variants consistently generated overt lung macro-metastases, whereas mice inoculated orthotopically with local neuroblastoma variants generated lung micro-metastases but no macro-metastases[18]. Both the lung macro-metastatic and micro-metastatic cells were cultured yielding macro-metastatic (MacroNB) and micro-metastatic neuroblastoma (MicroNB) cell variants. These variants share the same genetic background.

The MicroNB cells were found to express significantly higher levels of the MRD marker PHOX2B, compared with the MacroNB cells which express no or very low levels of PHOX2B. Further characterization of these variants revealed that the MacroNB cells express a more malignant phenotype than the MicroNB cells [18].

In this study we asked if PHOX2B is involved in shaping the malignant and metastatic phenotype of neuroblastoma cells. We also investigated the mechanism regulating $\mathrm{PHOX} 2 \mathrm{~B}$ expression in MicroNB and MacroNB cells.

\section{RESULTS}

\section{Downregulation of PHOX2B expression in MicroNB cells}

In a previous study we found that MicroNB cells, but not MacroNB cells, express high mRNA levels of the MRD marker PHOX2B [18]. In this work, we confirmed this finding at the mRNA level by qRT-PCR (Figure 1A) and at the protein level by western blot (Figure 1B). The qRT-PCR results showed that $\mathrm{PHOX} 2 \mathrm{~B}$ expression in the MicroNB cells was more than 4 orders of magnitude greater $(p<0.001)$ than in the MacroNB cells. Western blot analysis did not reveal any PHOX2B expression in the MacroNB cells $(\mathrm{p}<0.05)$.

To determine whether the differential expression of PHOX2B accounts for the differential malignant phenotype of MicroNB and MacroNB cells [18], we generated MicroNB cells in which PHOX2B expression was downregulated by PHOX2B specific shRNA (MicroNB-shPHOX2B). Control cells were infected with a non-silencing shRNA (MicroNBshControl). qRT-PCR assays showed that following infection with PHOX2B-specific shRNA, PHOX2B mRNA expression decreased almost four fold $(p<0.05)$ in the MicroNB-shPHOX2B cells (Figure 2A). Western blot analysis showed no expression of $\mathrm{PHOX} 2 \mathrm{~B}$ protein in the MicroNB-shPHOX2B cells $(\mathrm{p}<0.005)$ (Figure $2 \mathrm{~B})$.

\section{PHOX2B downregulation alters the expression of neuroblastoma-associated genes}

The influence of PHOX2B downregulation on the malignant phenotype of the cells was first evaluated by measuring expression levels of Tyrosine hydroxylase (TH) and GATA binding protein 3 (GATA3) which are genetically downstream to $\mathrm{PHOX} 2 \mathrm{~B}$ and have been linked with an unfavorable outcome and oncogenicity, respectively [19-23]. qRT-PCR measurements indicated that downregulation of PHOX2B significantly $(\mathrm{p}<0.05)$ increased TH expression. No significant change in GATA3 expression was observed (Figure 2C). The increased TH expression, a marker for bad prognosis in neuroblastoma $[22,24]$, suggested an involvement of PHOX2B in shaping the malignant phenotype of neuroblastoma cells.

\section{PHOX2B downregulation affects the malignant phenotype of the cells}

If expression levels of $\mathrm{PHOX} 2 \mathrm{~B}$ are indeed related to the malignant phenotype of neuroblastoma cells, it is to be expected that knockdown of PHOX2B would promote the malignant properties of these cells. This hypothesis was examined in the following set of experiments.

XTT-based viability assays were performed in order to determine the influence of PHOX2B downregulation on MicroNB cell viability. MicroNB-shControl and MicroNB-shPHOX2B cell viability was examined following $0,24,48$ and $72 \mathrm{hr}$ growth under normal conditions. Our results showed no significant difference in cell viability between the MicroNB-shControl and MicroNB-shPHOX2B cells (Figure 2D).

We previously found that MicroNB cells have a significantly lower migratory capacity than MacroNB cells [18]. Here, we examined whether the low migratory capacity of MicroNB cells is associated with the high PHOX2B expression levels of these cells. Using the wound healing assay it was found that PHOX2B knockdown significantly $(\mathrm{p}<0.05)$ impaired the ability of the cells to migrate and close the wound: only $32 \%, 47 \%$ and $61 \%$ wound closure at 24,48 and $72 \mathrm{hr}$ respectively was measured for the MicroNB-shPHOX2B cells, compared with 51\%, 71\% and 93\% closure measured for the MicroNB-shControl cells (Figure 2E). This result cannot be attributed to different proliferation rates of MicroNB-shPHOX2B and MicroNBshControl cells since no such difference was observed (Figure 2D). It shows, however, that $\mathrm{PHOX} 2 \mathrm{~B}$ restrains the migratory capacity of neuroblastoma cells.

\section{PHOX2B knockdown increases tumorigenicity and lung and bone-marrow metastasis}

To establish whether PHOX2B expression influences the tumorigenic and metastatic phenotype of MicroNB cells, we orthotopically inoculated the MicroNB-shControl and MicroNB-shPHOX2B cells to the adrenal gland of nude mice. Mice were weighed weekly. At the time of sacrifice, mice inoculated with MicroNBshPHOX2B cells weighed significantly more than mice inoculated with MicroNB-shControl cells $(\mathrm{p}<0.05)$ (Figure $3 \mathrm{~A})$; The increased weight of the mice inoculated with 
A.

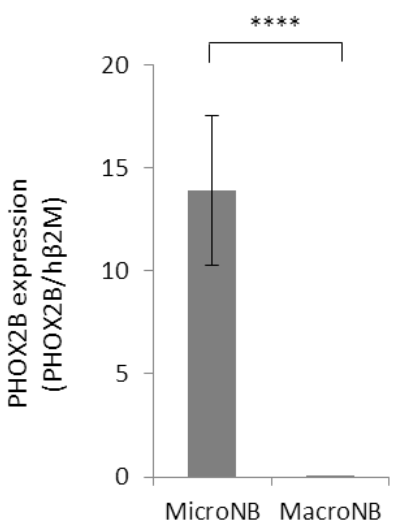

B.
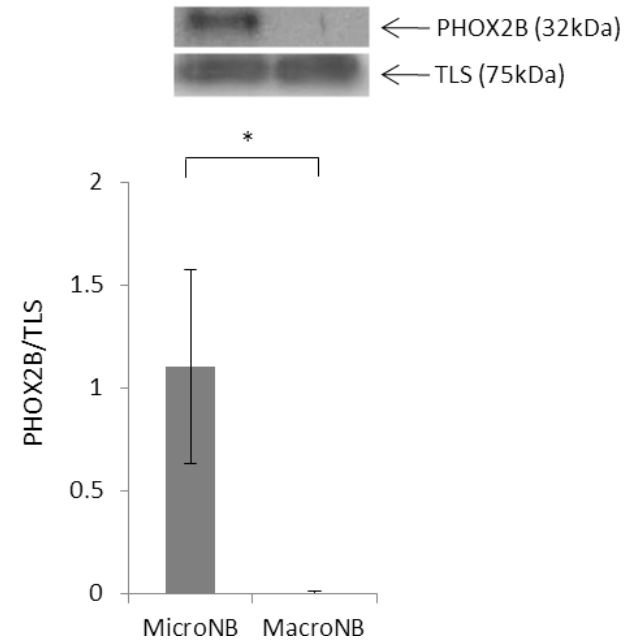

Figure 1: PHOX2B expression is higher in MicroNB than in MacroNB cells. PHOX2B mRNA and protein levels were examined in the MicroNB and MacroNB cells. A. PHOX2B mRNA level in the MicroNB and MacroNB cells was examined by qRT-PCR and normalized to human $\beta 2 \mathrm{M}$ expression B. Nuclear cell lysates of MacroNB and MicroNB cells were subjected to western blot analysis. Specific antibodies were used for protein identification: anti-PHOX2B and anti-TLS (used as loading control). PHOX2B protein level was calculated in reference to TLS, as measured by densitometry. The blot presents a representative experiment of three independent ones. Data represent the mean $\pm \mathrm{SD}$ of three independent experiments. Significance was evaluated using Student's $t$-test. ${ }^{*}{ }^{*} p<0.05,{ }^{* * * *}-p<0.001$.

A.

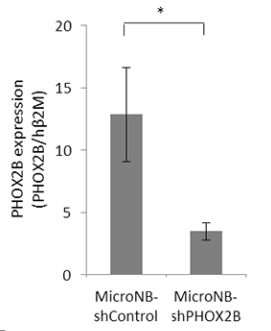

D.
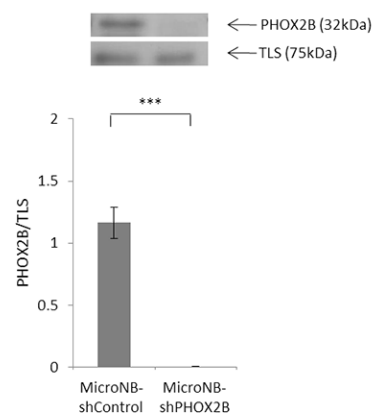

E.

c.
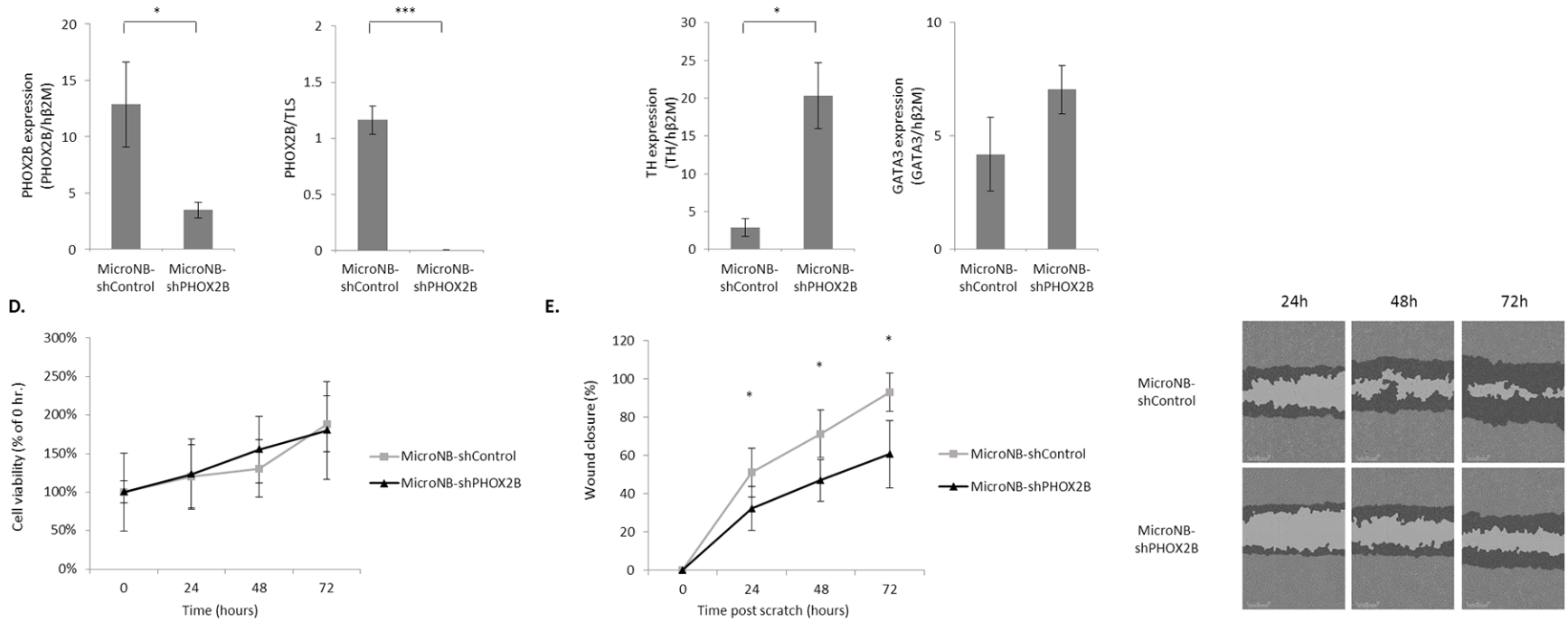

Figure 2: Downregulation of PHOX2B in MicroNB cells affects their malignant phenotype. Downregulation of $\mathrm{PHOX} 2 \mathrm{~B}$ in the MicroNB variant influences neuroblastoma-associated-genes expression and cell-migration, but not cell-viability. A. PHOX2B mRNA level in the MicroNB-shControl and MicroNB-shPHOX2B cells was examined by qRT-PCR and normalized to human $\beta 2 \mathrm{M}$. B. Nuclear cell lysates of MicroNB-shControl and MicroNB-shPHOX2B cells were subjected to western blot analysis. Specific antibodies were used for protein identification: anti-PHOX2B and anti-TLS (used as loading control). PHOX2B protein level was calculated in reference to TLS, as measured by densitometry. The blot presents a representative experiment of three independent ones. C. RNA extracted from MicroNB-shControl and MicroNB-shPHOX2B cells was used for qRT-PCR to measure changes in TH and GATA3 mRNA expression levels. All gene expression levels were normalized to human $\beta 2 \mathrm{M}$. D. MicroNB-shControl and MicroNB-shPHOX2B cells were examined for their viability by an XTT-based assay at 0, 24, 48 and 72hr following seeding. Cell viability at each time point is presented relative to cell viability at $0 \mathrm{hr}$. E. MicroNB-shControl and MicroNB-shPHOX2B cells were examined for their migratory capacity in a wound healing assay. Shown are representative photomicrographs of three independent experiments, taken at 24, 48 and $72 \mathrm{hr}$ post scratch. Image analysis was performed by IncuCyte software. Highlighted in black are cells that had migrated, and in light grey is the wound area that has not closed yet. Percentage of wound closure at every time point was calculated relatively to the wound width at $0 \mathrm{hr}$. Data represent the mean $\pm \mathrm{SD}$ of three independent experiments. Significance was evaluated using Student's $t$-test. ${ }^{*} p<0.05,{ }^{* * *-} p<0.005$. 
MicroNB-shPHOX2B cells was probably due to their heavier tumor and metastatic load (Figure 3B).

Local tumors, lungs and bone-marrow were evaluated for tumor load and PHOX2B expression. At the time of sacrifice, no overt lung macro-metastases were seen. A significant difference was observed in the local tumor size: Mice inoculated with MicroNB-shPHOX2B cells developed larger local tumors than MicroNB-shControl-inoculated mice (Figure 3B). qRT-PCR performed with RNA extracted from the local tumors, established that the tumors maintained the original PHOX2B expression of the injected cells: Cells harvested from local tumors of MicroNB-shControlinoculated mice expressed higher levels of PHOX2B than cells harvested from local tumors of MicroNB-shPHOX2Binoculated mice $(\mathrm{p}<0.05)$ (Figure $3 \mathrm{C})$.

Since no overt metastases were apparent at the time of sacrifice, a qRT-PCR was performed to evaluate the presence of micro-metastases in the lungs and bonemarrow of the inoculated mice. The results indicated that the knockdown of PHOX2B in MicroNB cells increased significantly their micro-metastatic load. A 50 and 40 fold higher load $(\mathrm{p}<0.05)$ was detected respectively in the lungs and bone-marrow of mice bearing MicroNB-shPHOX2B tumors than in the lungs and bone-marrow of control mice (Figure 3D, 3E). This result supports our previous finding that MacroNB cells expressing no or very low levels of PHOX2B, produce a higher metastatic burden than high PHOX2B expressing MicroNB cells [18]. More importantly, this indicates that $\mathrm{PHOX} 2 \mathrm{~B}$ is a regulator of neuroblastoma progression and metastasis.

\section{The mechanism regulating the differential expression of PHOX2B in MicroNB and MacroNB cells}

To investigate the mechanism responsible for the differential expression of PHOX2B in the MicroNB and MacroNB variants, we sequenced the PHOX2B gene and examined the role of methylation in $\mathrm{PHOX} 2 \mathrm{~B}$ gene expression, as detailed in the following set of experiments.

\section{PHOX2B sequence is identical in the MicroNB and MacroNB cells}

To exclude the possibility that heterozygous mutations of the PHOX2B gene [25] are the cause of the PHOX2B differential expression in the MicroNB and MacroNB cells, we sequenced the PHOX2B gene in these two cell variants. A 5754bp long fragment
A.

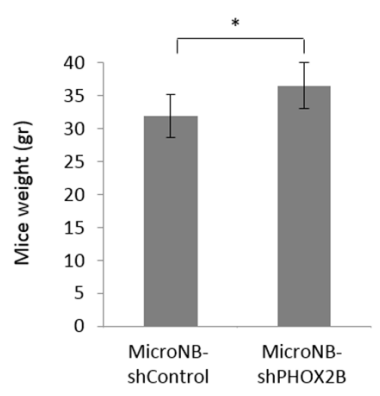

C.

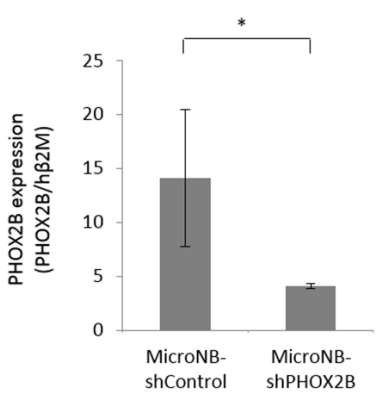

B.

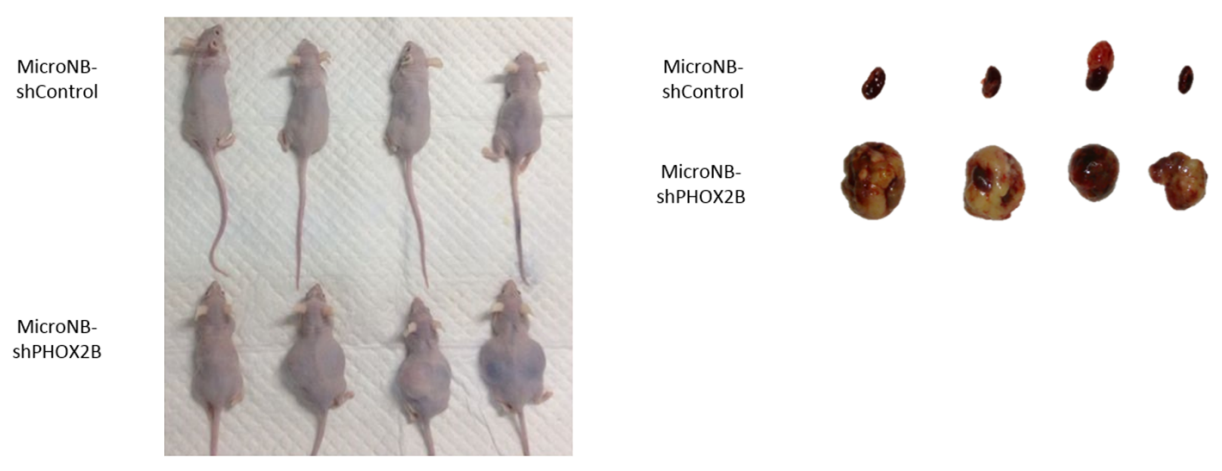

D.

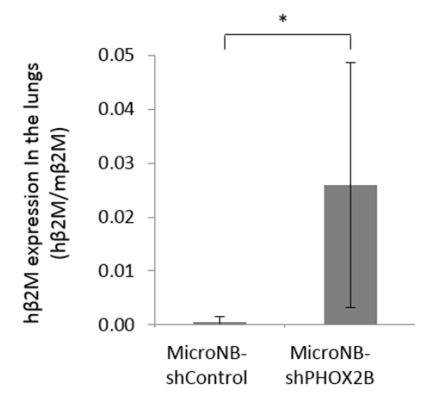

E.

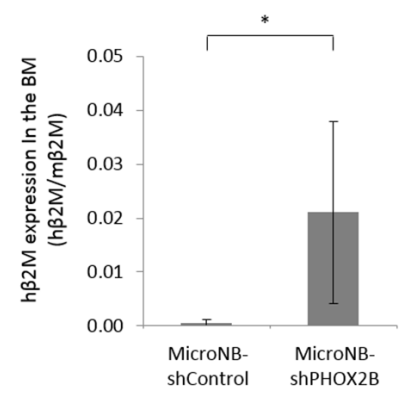

Figure 3: Downregulation of PHOX2B increases tumorigenicity and metastasis. Nude mice to which MicroNB-shControl and MicroNB-shPHOX2B cells were orthotopically inoculated were sacrificed when moribund, and primary tumors, bone-marrow and lungs were extracted for RNA quantitation. A. Mice were weighed weekly. Presented is the average weight of mice when sacrificed. B. A representative photo presenting mice and primary tumors, four of each group. C. RNA samples extracted from primary tumors were used to measure PHOX2B mRNA expression: PHOX2B mRNA levels were normalized to the human $\beta 2 \mathrm{M}$ expression levels. D, E. RNA samples extracted from lungs (D) and bone-marrow (E) were used to measure micro metastatic burden, by measuring human $\beta 2 \mathrm{M}$ mRNA expression level. Human $\beta 2 \mathrm{M}$ mRNA levels were normalized to the mouse $\beta 2 \mathrm{M}$ expression levels. Data represent the mean $(n=10,5$ mice in each group) $\pm \mathrm{SD}$. Significance was evaluated using Student's $t$-test. ${ }^{*} p<0.05$. 
containing the region spanning from the core PHOX2B promoter up to the end of exon 3 was isolated from the MicroNB and MacroNB cells by PCR and sequenced. It was found that both cells share an identical sequence. Also, two point mutations $(4181 \mathrm{~A} \rightarrow \mathrm{C}$ and $4194 \mathrm{C} \rightarrow \mathrm{T}$; numbered according to RefSeq accession no.: NG 008243.1) were found in the PHOX2B promoter region of both the MicroNB and the MacroNB cells. However, these mutations were neither located in a $\mathrm{CpG}$ dinucleotide or in a known transcription factor binding site [26, 27] (Figure 4A). This determined that the differential expression of PHOX2B in MicroNB and MacroNB cells is not derived from changes in its genomic sequence.

\section{The PHOX2B promoter is highly methylated in MacroNB cells}

PHOX2B promoter methylation leads to PHOX2B inactivation in neuroblastoma tumors and cell lines [28]. In order to establish whether promoter methylation is responsible for the differential expression of $\mathrm{PHOX} 2 \mathrm{~B}$ in MicroNB and MacroNB cells, we mapped the methylation pattern of the PHOX2B promoter in these cells. We first performed a bisulfite modification of the genome of the cells followed by PCR isolation of four amplicons containing fragments of the PHOX2B promoter (along 1061bp): amplicon I and II generated from the MicroNB converted genome, and amplicon I and II generated from the MacroNB

A.

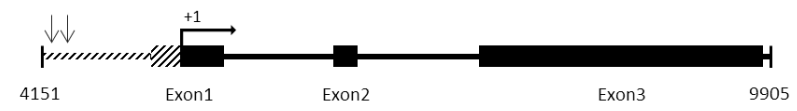

B.

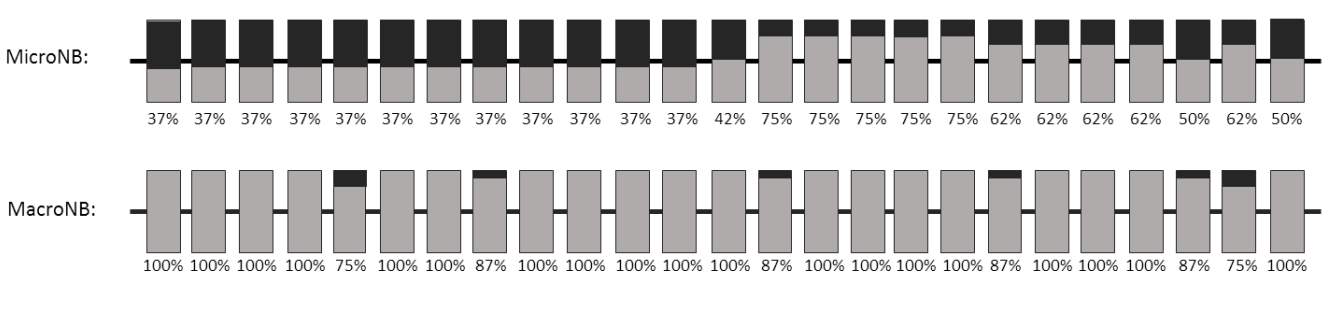

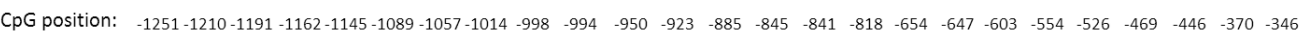

Methylated

Unmethylated

C.

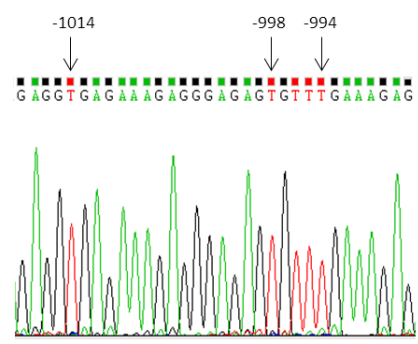

MicroNB

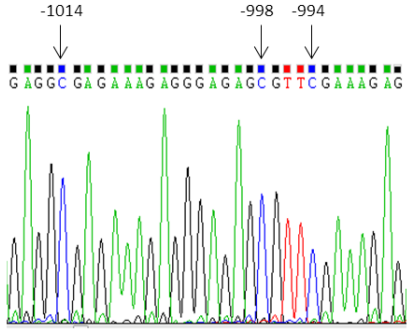

MacroNB

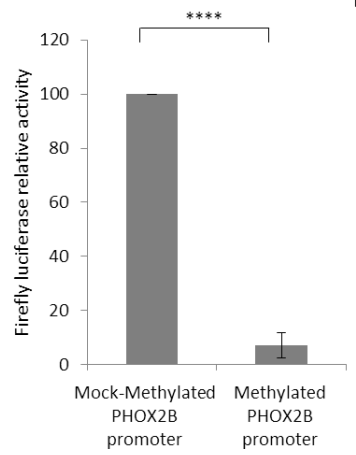

E.

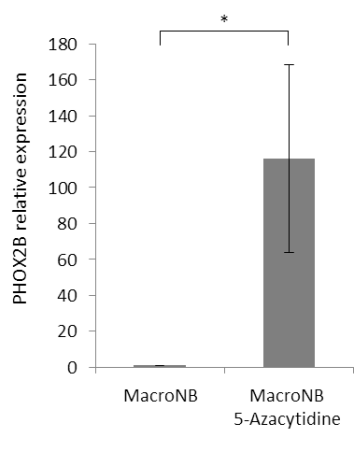

Figure 4: High methylation level of the PHOX2B promoter reduces its expression. The genomic sequence and promoter methylation pattern of PHOX2B gene, as well as the effect of promoter methylation on gene transcription, were examined in MicroNB and MacroNB cells. A. Genomic DNA extracted from MicroNB and MacroNB cells was used for sequencing of a 5754bp long fragment containing the region spanning from the core PHOX2B promoter up to the end of exon 3 (positions 4151-9905; NG_008243.1). Striped area marks the core PHOX2B promoter, arrows mark point mutations: $4181 \mathrm{~A} \rightarrow \mathrm{C}$ and $4194 \mathrm{C} \rightarrow \mathrm{T}$, the +1 arrow symbolizes the transcription start site [27]. B. Genomic DNA extracted from MicroNB and MacroNB cells was used in bisulfite sequencing analysis (25 CpG dinucleotides along a $1061 \mathrm{bp}$ sequence of the PHOX2B promoter). $\mathrm{CpG}$ positions are marked relatively to the +1 transcription start site [27]. Indicated below each $\mathrm{CpG}$ position is its methylation status. C. Representative sequencing results of the PHOX2B promoter in MicroNB and MacroNB cells following bisulfite conversion. D. PHOX2B Promoter activity was measured by a luciferase reporter assay. Firefly luciferase reporter gene activity was determined by transient transfection of SK-NMC cells with pGL2-PHOX2B-MOCK (Mock-Methylated PHOX2B promoter) or pGL2PHOX2B-MET (Methylated PHOX2B promoter) vectors. The graph shows Firefly luciferase activity after normalization to Renilla luciferase activity and relative to the activity measured in Mock-Methylated PHOX2B promoter. E. Following a 96hr treatment with the demethylation reagent 5-Azacytidine, PHOX2B mRNA expression in MacroNB cells and in MacroNB 5-Azacytidine treated cells was examined by qRT-PCR and normalized to RPS13 expression. The graph shows PHOX2B normalized expression relative to the expression measured in MacroNB cells. Results represent the mean \pm SD of three independent experiments. Significance was evaluated using Student's $t$-test. ${ }^{*} p<0.05$, ${ }^{* * * *}-p<0.001$. 
converted genome. Eight clones randomly selected from each amplicon were used to examine the methylation status (the percentage of methylation in each $\mathrm{CpG}$ dinucleotide) of the PHOX2B promoter in each variant. Sequencing analysis of these clones showed a 75-100\% methylation along 25 $\mathrm{CpG}$ dinucleotides in the $\mathrm{PHOX} 2 \mathrm{~B}$ promoter region of MacroNB cells compared with only $37-75 \%$ methylation of these $\mathrm{CpG}$ dinucleotides in MicroNB cells (Figure 4B). A representative sequencing result shows how three Cytosine nucleotides (CpG positions: -1014, -998 and -994) are unmethylated in MicroNB cells, thus appearing as Thymine in the DNA, while the same nucleotides are methylated in the MacroNB cells, thus appearing as Cytosine (Figure 4C). These results strongly suggest methylation as the cause for PHOX2B lack of expression in the MacroNB cells.

\section{In vitro methylation of the PHOX2B promoter diminishes transcription}

To further establish that methylation of the PHOX2B promoter is able to prevent gene transcription, we performed a luciferase reporter assay. The core $\mathrm{PHOX} 2 \mathrm{~B}$ promoter (a $1.3 \mathrm{~kb}$ sequence located upstream to the PHOX2B transcription start site) which was found to be sufficient for PHOX2B transcription [27] was cloned upstream to a Firefly luciferase gene in a pGL2 vector, to create a pGL2-PHOX2B vector. We then either methylated or mock-methylated the vector, and examined the Firefly luciferase transcription levels in transfected SK-NMC neuroblastoma cells. Renilla luciferase vector was used as an internal control. In cells transfected with the vector carrying the methylated promoter (Methylated PHOX2B promoter) 93\% less transcription $(\mathrm{P}<0.001)$ of the Firefly luciferase was detected, compared with transcription levels measured in cells transfected with the vector carrying the mock-methylated promoter (MockMethylated PHOX2B promoter) (Figure 4D). This result provides proof that methylation of the $\mathrm{PHOX} 2 \mathrm{~B}$ promoter is indeed capable of inhibiting gene transcription.

\section{Treatment with the demethylating agent 5-Azacytidine induces PHOX2B expression}

The demethylation reagent 5-Azacytidine incorporates into the DNA and inhibits DNA methylation [29]. We treated MacroNB cells, which are highly methylated in the PHOX2B promoter area (Figure 4B), with 5-Azacytidine for $96 \mathrm{hr}$ and measured PHOX2B mRNA levels by qRT-PCR. We found that following treatment, the relative PHOX2B mRNA expression was 100 fold elevated (Figure 4E), a direct evidence that PHOX2B expression in MacroNB cells is suppressed by methylation.

\section{DISCUSSION}

PHOX2B, a transcription factor and a specific and sensitive bio-marker for MRD in neuroblastoma patients $[11,12]$, is highly expressed in micro-metastatic neuroblastoma cells (MicroNB), but not, or significantly less in macro-metastatic cells (MacroNB). These cells also differ in their malignant and metastatic phenotype; the latter cells form lung macro-metastases, while the MicroNB cells generate only lung micro-metastases [18]. This study is based on the well-established fact that overt metastasis in a specific organ develops from precursor micro-metastatic cells residing in it [10, 30-34]. The main goal of this research was, therefore, to find out whether PHOX2B is functionally involved in shaping the micrometastatic phenotype of neuroblastoma cells.

In the present study we established that PHOX2B functions as a suppressor of neuroblastoma metastasis: The knockdown of PHOX2B in human neuroblastoma micrometastatic cells increased the tumorigenic and metastatic potential of the cells in orthotopically inoculated nude mice. As reported above, the PHOX2B-mediated downregulation induced an upregulation of $\mathrm{TH}$, a marker for bad prognosis in neuroblastoma [22]. It is still to be determined whether the upregulation of $\mathrm{TH}$ is involved in promoting the malignant behavior of neuroblastoma cells.

There was a discrepancy between the in vivo results indicating that the knockdown of PHOX2B promoted local tumor formation and increased metastatic load, and the in vitro results demonstrating that $\mathrm{PHOX} 2 \mathrm{~B}$ knockdown did not affect proliferation and decreased the migratory capacity of the cells. Discrepancies between in vivo and in vitro findings are rather common [35-38] and are to be expected. Tumors do not proliferate or progress without cross-talk with various types of stromal cells and their products $[39,40]$. Tumorigenesis and metastasis of neuroblastoma cells in vivo are regulated by a balanced function of positive and negative signals [10] including those regulated by $\mathrm{PHOX} 2 \mathrm{~B}$. The in vitro milieu, in which proliferation and migration of shPHOX2B neuroblastoma cells were measured, did not provide these additional signals.

Tumor progression is largely regulated by the tumor microenvironment [30, 41-43] and different microenvironments differently control gene expression of tumor cells [44-46]. Since the MicroNB and MacroNB variants share a common genetic background but initially originated from different microenvironments (the MicroNB cells originated from adrenal tumors and the MacroNB cells originated from lung metastasis) [17, 18], it is not unlikely that the differential expression of PHOX2B in these cells is due to genetic or epigenetic events that took place in the different microenvironments from which these variant progenitors were generated.

DNA hyper-methylation is an epigenetic event occurring in many cancer types [47-50] and one that plays a role in $\mathrm{PHOX} 2 \mathrm{~B}$ silencing in neuroblastoma cell-lines and tumors [28]. We found that the PHOX2B promoter, that has an identical sequence in MicroNB and MacroNB cells, is more highly methylated in the MacroNB cells 
than in the MicroNB cells. It was also established that methylation of the PHOX2B promoter is able to reduce transcription by $93 \%$. Treatment with the demethylating agent 5-azacytidine induced PHOX2B expression in the MacroNB cells, verifying that the low PHOX2B expression in the MacroNB cells is indeed due to high methylation of its gene in these cells.

Taken together this pre-clinical study demonstrates that PHOX2B is a metastasis suppressor, able to inhibit the tumorigenic and metastatic abilities of neuroblastoma cells and that this inhibitory function is likely mediated by the tumor microenvironment via an epigenetic mechanism.

\section{MATERIALS AND METHODS}

\section{Cell culture}

The human neuroblastoma lung micro-metastatic (MicroNB) and macro-metastatic (MacroNB) variants were generated using a mouse model for human neuroblastoma metastasis $[17,18]$ from the parental cell line MHH-NB-11 [16] and were maintained in culture as previously described [17]. SK-NMC cells were maintained in culture as previously described [51]. MicroNB-shControl and MicroNB-shPHOX2B cells were maintained as monolayer cultures in growth medium: RPMI 1640 medium supplemented with 10\% fetal calf serum (FCS), $100 \mathrm{U} / \mathrm{ml}$ streptomycin, $12.5 \mathrm{U} /$ $\mathrm{ml}$ nystatin, $100 \mathrm{U} / \mathrm{ml}$ penicillin, $2 \mathrm{mM}$ L-glutamine (all materials were purchased from Biological Industries, Beit Ha'emek, Israel), and $2 \mu \mathrm{g} / \mathrm{ml}$ puromycin (InvivoGen, San Diego, CA, USA). The cultures were incubated at $37^{\circ} \mathrm{C}$ in a mixture of $6.5 \%$ carbon dioxide. Cell authentication was performed by short tandem repeat analysis of DNA using the Type-it Microsatellite PCR Kit (Qiagen, Valencia, CA, USA), for the genes published by the ATCC (Manassas, VA, USA). All cultures were periodically examined for mycoplasma contamination.

\section{Animals}

Male athymic nude mice (BALB/c background) were purchased from Harlan Laboratories (Jerusalem, Israel). The mice were housed and maintained as previously described [52]. All experiments involving animals were approved by the TAU Institutional Animal Care and Use Committee. Mice of 7-10 weeks old were used for experiments in accordance with institutional ethical guidelines.

\section{qRT-PCR}

Total RNA was extracted from cell cultures using the EZ-RNA Total RNA Isolation Kit (Biological Industries) and used in quantitative real-time PCR (qRTPCR) as previously described [18]. From harvested organs, total RNA was extracted using the Bullet blender bead lysis kit (Next advance Inc., Averill Park, NY, USA) and the EZ-RNA Total RNA Isolation Kit (Biological Industries). Detection of human neuroblastoma cells (micro-metastases) in mouse tissue was performed as previously described [18], using specific primers for the human/mouse $\beta 2$ microglobulin. Primers used for mRNA amplification were: human $\beta 2$ microglobulin (NM_004048) For-5'-ATGTAAGCAGCATCATGGAG-3', Rev-5'-AAGCAAGCAGAATTTGGAAT-3'; mouse $\beta 2$ microglobulin (NM_009735.3) For-5'-CTGGTCTTTC TGGTGCTTGT-3', Rev- 5'-GGCGTGAGTATACTTGA ATTTGAG-3'; PHOX2B (NM_003924.3) For-5'-TACG CCGCAGTTCCTTACAA-3', Rev-5'-GAAGACCCTT TCCAGCTCTTT-3'; TH (NM_000360.3) For-5'-TGT ACTGGTTCACGGTGGAGTT-3', Rev-5'-AATCTCA GGCTCCTCAGACA-3'; GATA3 (NM_001002295.1) For-5'-ACACTCTGGAGGAGGAATGC-3', Rev-5'-CTG GATGCCTTCCTTCTTCATA-3'; RPS13 (NM_001017.2) For-5'-CGAAAGCATCTTGAGAGGAACA-3', Rev-5'TCGAGCCAAACGGTGAATC-3'.

\section{Western blotting}

Neuroblastoma cells were seeded in $60 \mathrm{~mm}$ plates $\left(1 \times 10^{6}\right.$ cells/plate) in normal growth conditions. $48 \mathrm{hr}$ following seeding, cells were washed twice with PBSx1 (Biological Industries) and stored in $-70^{\circ} \mathrm{c}$ until use. Cell pellets were lysed with buffer lysis (5mM Hepes $\mathrm{pH} 7.9,1 \mathrm{mM} \mathrm{Na} 3 \mathrm{VO} 4,1 \mathrm{mM} \mathrm{NaPPi}$, $1 \mathrm{mM} \mathrm{NaF}, 1 \mathrm{mM}$ PMSF, $2 \mu \mathrm{g} / \mathrm{ml}$ Leupeptin, $2 \mu \mathrm{g} / \mathrm{ml}$ Aprotinin, 1\% NP-40) then centrifuged for $20 \mathrm{~min}$ in $16,000 \mathrm{~g} 4^{\circ} \mathrm{c}$. For nuclear fractionation, the upper layer was removed and the remaining pellet was washed (20mM Hepes $\mathrm{pH} 7.9,5 \mathrm{mM} \mathrm{KCl}, 150 \mathrm{mM} \mathrm{NaCl}$ ), then centrifuged for $10 \mathrm{~min}$ in $16,000 \mathrm{~g}, 4^{\circ} \mathrm{c}$. The upper layer was removed and the remaining pellet was lysed with buffer lysis (20mM Hepes pH 7.9, 420mM NaCl, $1 \mathrm{mM}$ EDTA, 1mM EGTA, 1 $\mathrm{mM}$ dithiothreitol, $1 \mathrm{mM}$ PMSF, $1 \mathrm{mM}$ Na3VO4, $4 \mathrm{mM} \beta$ glycerol phosphate, $2 \mu \mathrm{g} / \mathrm{ml}$ Leupeptin, $2 \mu \mathrm{g} / \mathrm{ml}$ Aprotinin), then ice incubated for $30 \mathrm{~min}$ and centrifuged for $10 \mathrm{~min}$ in $16,000 \mathrm{~g}, 4^{\circ} \mathrm{c}$. The upper layer was used as the nuclear lysate, and protein concentration in the samples was determined by BCA Protein Assay reagent (Thermo Fisher Scientific, Waltham, MA, USA). Samples were subjected to western blot analysis as previously described [18]. Goat polyclonal anti-PHOX2B (1:200, Santa Cruz Biotechnology Inc., Santa Cruz, CA, USA) and Rabbit polyclonal anti-TLS/FUS (1:30,000, Abcam, Cambridge, UK) were used in PHOX2B and TLS detection, respectively. HRP-conjugated donkey antigoat IgG and HRP-conjugated goat anti-rabbit IgG (Jackson immunosearch laboratories, West Grove, PA, USA) were used as secondary antibodies, respectively, according to the manufacturer's instructions. 


\section{Downregulation of PHOX2B expression}

The downregulation of PHOX2B was constructed using pGIPZ vectors (Thermo Fisher Scientific). Seven different $p$ GIPZ vectors containing seven different shRNA sequences targeting PHOX2B mRNA (NM_003924.3) were examined for their downregulation efficiency using qRT-PCR and western blotting. Two of the seven vectors which showed the strongest effect (RHS443098843809, RHS4430-101168343) were then used for PHOX2B downregulation in the MicroNB variant (MicroNB-shPHOX2B). A sh-non-silencing pGIPZ vector (RHS4531) was used as a negative control (MicroNBshControl).

To produce the infectious viruses, the 293T packaging cell line was co-transfected using a calcium phosphate method with the lentiviral backbone plasmids shPHOX2B-pGIPZ or the sh-non-silencing-pGIPZ, packaging plasmid $\mathrm{pCMV} \triangle \mathrm{R} 8.2$ and envelope plasmid pVSV-G. After $48 \mathrm{hr}$, the virus particles in the medium were collected and filtrated $(0.45 \mathrm{~mm}$, Whatman $\mathrm{GmbH}$, Freiburg, Germany). A total of $1 \times 10^{6}$ MicroNB cells, seeded $24 \mathrm{hr}$ before infection, were infected overnight in the presence of $8 \mu \mathrm{g} / \mathrm{ml}$ polybrene and the virus-containing medium, which was afterwards replaced with fresh growth medium. After $72 \mathrm{hr} 2 \mu \mathrm{g} / \mathrm{ml}$ puromycin (InvivoGen) was added for additional 7 days to select stably infected cell population. After selection, puromycin was continuously added to the culture.

\section{In vitro viability assay}

MicroNB-shControl and MicroNB-shPHOX2B cells were seeded $\left(1 \times 10^{4}\right.$ per well) in a 96-well, flatbottomed, tissue culture plate. Viability under normal growth conditions [17] was monitored in triplicates using an XTT-based viability assay after $0,24,48$, and 72 hours, according to the manufacturer's instructions (Biological Industries). Cell viability was determined as previously described [18].

\section{Wound healing assay}

MicroNB-shControl and MicroNB-shPHOX2B cells were seeded onto a 96-well ImageLock tissue culture plate (Essen BioScience, Ann Arbor, MI, USA) which was pre-coated with $0.015 \%$ Poly-L-lysin (SigmaAldrich Corp., St Louis, MO, USA). Cells were allowed to attach over a $24 \mathrm{hr}$ period, then wounds were made with the 96-well WoundMaker (Essen BioScience). The culture plate was washed once with PBSx1 to remove detached cells, and a fresh growth medium was added. Images of the wounds were automatically acquired within the $\mathrm{CO}_{2}$ incubator by IncuCyte zoom (Essen BioScience). Typical photomicrographs were taken at $2 \mathrm{hr}$ intervals for a $72 \mathrm{hr}$ period. The data were analyzed with respect to wound confluence and calculated using the IncuCyte software (Essen BioScience).

\section{Orthotopic inoculation of tumor cells}

An orthotopic inoculation to the adrenal gland of athymic nude mice was performed with $1 \times 10^{6}$ MicroNBshControl or MicroNB-shPHOX2B cells suspended in $50 \mu$ growth medium (RPMI-1640 containing 5\% fetal calf serum (FCS); Biological Industries). The intra-adrenal inoculation required surgical exposure of the left adrenal gland under anesthesia and was performed as previously described [17]. We weekly monitored local tumor development and mice weights. Mice were sacrificed when moribund (about 90 days after inoculation) and local adrenal tumor, lungs and sternum (bone marrow) were harvested. The organs were immediately flash-frozen on dry ice, then stored at $-70^{\circ} \mathrm{c}$ until used for RNA extraction.

\section{Sequencing of the PHOX2B gene}

Genomic DNA was extracted from the MicroNB and MacroNB cells using QIAamp DNA Mini Kit (Qiagen). We custom designed primers covering a 5754bp sequence containing the region spanning from the core PHOX2B promoter up to the end of exon 3 (positions 4151- 9905; NG_008243.1). The primers for amplicon I are: For- 5'-TACAGTCCGCAAACCTAAAAGG-3', Rev- 5'-CTAAGCTTTACGTCTCATCGCA-3', for amplicon II: For- 5'-TACGCCGCAGGTAAGGACC-3', Rev- 5'-GTCAGTGCTCTTGGCCTCTT-3', for amplicon III: For- 5'-GCAAAGAGGCCAAGAGCACTGA-3', Rev5'-TTATCAATGCCCTGGTGTGCTTCT-3'. Amplicons were PCR amplified using the KAPA HiFi PCR Kit (Kapa Biosystems, Boston, MA, USA) and sequenced in the ABI 3500xl Genetic analyzer (Life technologies, Waltham, MA, USA). PCR conditions were: 39 cycles of: $98^{\circ} \mathrm{c}$ for $20 \mathrm{sec}, 68^{\circ} \mathrm{c} / 69^{\circ} \mathrm{c} / 68^{\circ} \mathrm{c}$ for $15 \mathrm{sec}, 72^{\circ} \mathrm{c}$ for $1.50 \mathrm{~min} / 1.20 \mathrm{~min} / 3.40 \mathrm{~min}$, for amplicons I, II and III respectively.

\section{Bisulfite conversion and methylation pattern analysis of the PHOX2B promoter}

Genomic DNA was extracted from the MicroNB and MacroNB cells using QIAamp DNA Mini Kit (Qiagen). 500ng of sample DNA was treated with sodium bisulfite using the EZ DNA Methylation-lightning Kit (Zymo Research, Irvine, CA, USA). Treatment was according to the manufacturer's instructions, only that incubation with L-Desulphonation Buffer was performed in $30^{\circ} \mathrm{c}$. We custom designed primers for two amplicons spanning the core PHOX2B promoter, covering a $1061 \mathrm{bp}$ long sequence in total, starting $1322 \mathrm{bp}$ upstream to the first transcription start site [27]. The primers for amplicon I are: For- 5'-AGAGGAAGGATTTAATGAAATAGT-3' and 
Rev- 5'-CCRTTATATTCTTTACTCTAATTCTATCTAT TTAC-3'. For amplicon II: For- 5'-TTGYGTTAATG TAAATAGATAGAATTGAGAG-3' and Rev- 5'-CACCR AACCCCTAATCCTCCCTTCTAACCAAC-3'. These primers were used in PCR with GoTaq DNA polymerase (Promega, Madison, WI, USA), with $3 \mathrm{mM} \mathrm{Mg}^{2+}$ and an annealing temp of $56^{\circ} \mathrm{c}$. Thus we generated four PCR products: amplicon I and II generated from the MicroNB converted genome, and amplicon I and II generated from the MacroNB converted genome. Each PCR product was cloned into the pCRII-TOPO vector (Invitrogene, Waltham, MA, USA), then transformed to XL1 blue bacteria and grown on an Amp+Tet agarose plates. Eight clones were randomly selected from each of the PCR products and sequenced in the ABI 3500xl Genetic analyzer (Life technologies) to detect methylation level of each $\mathrm{CpG}$ dinucleotides. Sequencing results data were analyzed by the Chromas LITE software (Technelysium, South Brisbane, Australia).

\section{Luciferase assay}

A 1300bp amplicon containing the core PHOX2B promoter (positions 3948- 5245; NG_ 008243.1) was isolated from the MacroNB variant by DNA extraction using the QIAamp DNA Mini Kit (Qiagen) followed by PCR using the KAPA HiFi PCR Kit (Kapa Biosystems). Forward and reverse primers contained either a SacI or Bgl2 recognition sites, respectively (For- 5'-ATATATGAGCTCTTCCCTTAT TCTGCTAGGGCCTAA-3', Rev- 5'-AATAATAGATCT CCTTACTACCAGCAGAGCCTAGTTT-3'). PCR conditions were: 2 cycles of: $98^{\circ} \mathrm{c}$ for $30 \mathrm{sec}, 60^{\circ} \mathrm{c}$ for $15 \mathrm{sec}, 72^{\circ} \mathrm{c}$ for $1.30 \mathrm{~min}$ followed by 43 cycles of: $98^{\circ} \mathrm{c}$ for $30 \mathrm{sec}, 68^{\circ} \mathrm{c}$ for $15 \mathrm{sec}, 72^{\circ} \mathrm{c}$ for $1.5 \mathrm{~min}$. The amplicon was cloned into a pGL2-basic vector (promega) which contains luciferase as a reporter gene. The vector was then either methylated/ mock-methylated by SssI methylase (New England Biolabs, Ipswich, MA, USA) thus generating a pGL2-PHOX2B-MET or pGL2-PHOX2BMOCK vector, respectively. The procedure for mockmethylation was identical except no SssI methylase was included. SK-NMC cells were seeded in a 24 well plate, allowed to attach over a $24 \mathrm{hr}$ period, and transfected with either pGL2-PHOX2B-MET or a pGL2-PHOX2BMOCK vectors, and with the Renilla luciferase pRL-TK vector (promega). Transfection was performed O.N. using Lipofectamin 2000 and OptiMem reduced serum medium (invitrogne). 24hr following transfection SK-NMC-pGL2PHOX2B-MET and SK-NMC-pGL2-PHOX2B-MOCK cells were removed by Trypsin-B (Biological Industries), washed twice with PBSx1 (Biological Industries) and pellets were stored in $-70^{\circ} \mathrm{c}$. Pellets were subjected to luciferase assay using the Dual-Luciferase Reporter Assay System (promega) in the Veritas microplate luminometer (Turnerbiosystems, Sunnyvale, CA, USA). Results were then analyzed with the Veritas Microplate Luminometer software (Turnerbiosystems).

\section{5-Azacytidine treatment}

MacroNB cells were seeded in $60 \mathrm{~mm}$ plates $\left(7 \times 10^{5}\right.$ cells/plate) and allowed to attach over a $24 \mathrm{hr}$ period. Cells were then treated with $5 \mu \mathrm{M}$ (dissolved in $50 \%$ acetic acid) of the demethylation reagent 5-Azacytidine (SigmaAldrich Corp.) for $72 \mathrm{hr}$, then growth medium was replaced with fresh medium and $5 \mu \mathrm{M}$ of 5-Azacytidine were added for another $24 \mathrm{~h}$ period. After the $96 \mathrm{hr}$ treatment RNA was extracted from the cells and qRT-PCR was performed (see qRT-PCR in this section).

\section{Statistical analysis}

Paired or unpaired Student's $t$-test was used to compare in vitro and in vivo results.

\section{ACKNOWLEDGMENTS}

The authors thank Dr. Mickey Harlev and Dr. Maya Levin Arama (Animal Care Facilities, Sackler Faculty of Medicine, Tel-Aviv University), for the help with animal experiments. We also thank Prof. Eran Bacharach (The George S. Wise Faculty of Life Sciences, Tel-Aviv University) and Dr. Noam Shomron and Ronna Shayevitch (Sackler Faculty of Medicine, Tel-Aviv University) for useful discussions. This research was supported by the German Research Foundation (Deutche Forschungsgemeinschaft DFG), the James \& Rita Leibman Endowment Fund for Cancer Research and by the Fred August and Adele Wolpers Charitable Fund and the Sara and Natan Blutinger Foundation. The authors disclose no potential conflicts of interest.

\section{CONFLICTS OF INTEREST}

The authors declare no conflict of interest.

\section{GRANT SUPPORT}

This research was supported by the German Research Foundation (Deutche Forschungsgemeinschaft DFG) grant BA4027/6-1 (to IPW), by the James \& Rita Leibman Endowment Fund for Cancer Research (to IPW) and by the Fred August and Adele Wolpers Charitable Fund (Clifton, NJ, USA) and the Sara and Natan Blutinger Foundation (West Orange, NJ, USA).

\section{REFERENCES}

1. Brodeur GM. Neuroblastoma: biological insights into a clinical enigma. Nature reviews Cancer. 2003; 3:203-216.

2. Maris JM and Matthay KK. Molecular biology of neuroblastoma. Journal of clinical oncology. 1999; 17:2264-2279. 
3. Kammen BF, Matthay KK, Pacharn P, Gerbing R, Brasch $\mathrm{RC}$ and Gooding CA. Pulmonary metastases at diagnosis of neuroblastoma in pediatric patients: CT findings and prognosis. American journal of roentgenology. 2001; 176:755-759.

4. Westermann F and Schwab M. Genetic parameters of neuroblastomas. Cancer letters. 2002; 184(2):127-147.

5. Burchill SA. Micrometastases in neuroblastoma: are they clinically important? Journal of clinical pathology. 2004; 57:14-20.

6. Moss TJ, Reynolds CP, Sather HN, Romansky SG, Hammond GD and Seeger RC. Prognostic value of immunocytologic detection of bone marrow metastases in neuroblastoma. The New England journal of medicine. 1991; 324:219-226.

7. Pantel K and Alix-Panabieres C. The clinical significance of circulating tumor cells. Nature clinical practice Oncology. 2007; 4:62-63.

8. Wikman H, Vessella R and Pantel K. Cancer micrometastasis and tumour dormancy. APMIS. 2008; 116:754-770.

9. Cheung IY, Feng Y, Vickers A, Gerald W and Cheung NK. Cyclin D1, a novel molecular marker of minimal residual disease, in metastatic neuroblastoma. The Journal of molecular diagnostics. 2007; 9:237-241.

10. Maman S, Witz, IP. (2013). The metastatic microenvironment. In: Shurin MR, Umansky, V, Malyguine, A, ed. The Tumor Immunoenvironment: Springer: N.Y, pp. 15-38.

11. Stutterheim J, Gerritsen A, Zappeij-Kannegieter L, Kleijn I, Dee R, Hooft L, van Noesel MM, Bierings M, Berthold F, Versteeg R, Caron HN, van der Schoot CE and Tytgat GA. PHOX2B is a novel and specific marker for minimal residual disease testing in neuroblastoma. Journal of clinical oncology. 2008; 26:5443-5449.

12. Huber K, Karch N, Ernsberger U, Goridis C and Unsicker $\mathrm{K}$. The role of Phox2B in chromaffin cell development. Developmental biology. 2005; 279:501-508.

13. Trochet D, Bourdeaut F, Janoueix-Lerosey I, Deville A, de Pontual L, Schleiermacher G, Coze C, Philip N, Frebourg T, Munnich A, Lyonnet S, Delattre O and Amiel J. Germline mutations of the paired-like homeobox 2B (PHOX2B) gene in neuroblastoma. American journal of human genetics. 2004; 74:761-764.

14. Mosse YP, Laudenslager M, Khazi D, Carlisle AJ, Winter CL, Rappaport E and Maris JM. Germline PHOX2B mutation in hereditary neuroblastoma. American journal of human genetics. 2004; 75:727-730.

15. Abel F, Dalevi D, Nethander M, Jornsten R, De Preter K, Vermeulen J, Stallings R, Kogner P, Maris J and Nilsson S. A 6-gene signature identifies four molecular subgroups of neuroblastoma. Cancer cell international. 2011; 11:9.

16. Pietsch T, Gottert E, Meese E, Blin N, Feickert HJ, Riehm $\mathrm{H}$ and Kovacs $\mathrm{G}$. Characterization of a continuous cell line (MHH-NB-11) derived from advanced neuroblastoma. Anticancer research. 1988; 8:1329-1333.
17. Nevo I, Sagi-Assif O, Edry Botzer L, Amar D, Maman S, Kariv N, Leider-Trejo LE, Savelyeva L, Schwab M, Yron I and Witz IP. Generation and characterization of novel local and metastatic human neuroblastoma variants. Neoplasia. 2008; 10:816-827.

18. Edry Botzer L, Maman S, Sagi-Assif O, Meshel T, Nevo I, Bauerle T, Yron I and Witz IP. Lung-residing metastatic and dormant neuroblastoma cells. The American journal of pathology. 2011; 179:524-536.

19. Pattyn A, Morin X, Cremer H, Goridis C and Brunet JF. The homeobox gene Phox $2 \mathrm{~b}$ is essential for the development of autonomic neural crest derivatives. Nature. 1999; 399:366-370.

20. Stanke M, Junghans D, Geissen M, Goridis C, Ernsberger $\mathrm{U}$ and Rohrer H. The Phox2 homeodomain proteins are sufficient to promote the development of sympathetic neurons. Development. 1999; 126:4087-4094.

21. Tsarovina K, Pattyn A, Stubbusch J, Muller F, van der Wees J, Schneider C, Brunet JF and Rohrer H. Essential role of Gata transcription factors in sympathetic neuron development. Development. 2004; 131:4775-4786.

22. Trager C, Kogner P, Lindskog M, Ponthan F, Kullman A and Kagedal B. Quantitative analysis of tyrosine hydroxylase mRNA for sensitive detection of neuroblastoma cells in blood and bone marrow. Clin Chem. 2003; 49:104-112.

23. Molenaar JJ, Ebus ME, Koster J, Santo E, Geerts D, Versteeg R and Caron HN. Cyclin D1 is a direct transcriptional target of GATA3 in neuroblastoma tumor cells. Oncogene. 2010; 29:2739-2745.

24. Viprey VF, Gregory WM, Corrias MV, Tchirkov A, Swerts K, Vicha A, Dallorso S, Brock P, Luksch R, Valteau-Couanet D, Papadakis V, Laureys G, Pearson AD, Ladenstein R and Burchill SA. Neuroblastoma mRNAs predict outcome in children with stage 4 neuroblastoma: a European HR-NBL1/SIOPEN study. Journal of clinical oncology. 2014; 32:1074-1083.

25. Trochet D, Hong SJ, Lim JK, Brunet JF, Munnich A, Kim KS, Lyonnet S, Goridis C and Amiel J. Molecular consequences of PHOX2B missense, frameshift and alanine expansion mutations leading to autonomic dysfunction. Human molecular genetics. 2005; 14:3697-3708.

26. Cargnin F, Flora A, Di Lascio S, Battaglioli E, Longhi R, Clementi $\mathrm{F}$ and Fornasari D. PHOX2B regulates its own expression by a transcriptional auto-regulatory mechanism. The Journal of biological chemistry. 2005; 280:37439-37448.

27. Jong Hong S, Chae $\mathrm{H}$ and Kim KS. Molecular cloning and characterization of the promoter region of the human Phox $2 \mathrm{~b}$ gene. Brain research Molecular brain research. 2004; 125:29-39.

28. de Pontual L, Trochet D, Bourdeaut F, Thomas S, Etchevers H, Chompret A, Minard V, Valteau D, Brugieres L, Munnich A, Delattre O, Lyonnet S, Janoueix-Lerosey I and Amiel J. Methylation-associated PHOX2B gene silencing is 
a rare event in human neuroblastoma. European journal of cancer. 2007; 43:2366-2372.

29. Christman JK. 5-Azacytidine and 5-aza-2'-deoxycytidine as inhibitors of DNA methylation: mechanistic studies and their implications for cancer therapy. Oncogene. 2002; 21:5483-5495.

30. Klein-Goldberg A, Maman S and Witz IP. The role played by the microenvironment in site-specific metastasis. Cancer letters. 2014; 352:54-58.

31. Chambers AF, Groom AC and MacDonald IC. Dissemination and growth of cancer cells in metastatic sites. Nat Rev Cancer. 2002; 2:563-572.

32. Balic M, Williams A, Dandachi $\mathrm{N}$ and Cote RJ. Micrometastasis: detection methods and clinical importance. Cancer Biomark. 2010; 9:397-419.

33. Riethdorf S, Wikman H and Pantel K. Review: Biological relevance of disseminated tumor cells in cancer patients. International journal of cancer. 2008; 123:1991-2006.

34. Alix-Panabieres C, Riethdorf S and Pantel K. Circulating tumor cells and bone marrow micrometastasis. Clin Cancer Res. 2008; 14:5013-5021.

35. Vatner RE and Formenti SC. Myeloid-derived cells in tumors: effects of radiation. Semin Radiat Oncol. 2015; 25:18-27.

36. Langenkamp E, Kamps JA, Mrug M, Verpoorte E, Niyaz Y, Horvatovich P, Bischoff R, Struijker-Boudier H and Molema $\mathrm{G}$. Innovations in studying in vivo cell behavior and pharmacology in complex tissues--microvascular endothelial cells in the spotlight. Cell Tissue Res. 2013; 354:647-669.

37. Bjarnsholt $\mathrm{T}$, Alhede M, Alhede M, Eickhardt-Sorensen $\mathrm{SR}$, Moser C, Kuhl M, Jensen PO and Hoiby N. The in vivo biofilm. Trends Microbiol. 2013; 21:466-474.

38. Sung JH, Esch MB, Prot JM, Long CJ, Smith A, Hickman JJ and Shuler ML. Microfabricated mammalian organ systems and their integration into models of whole animals and humans. Lab Chip. 2013; 13:1201-1212.

39. Witz IP. Tumor-microenvironment interactions: dangerous liaisons. Advances in cancer research. 2008; 100:203-229.

40. Witz IP and Levy-Nissenbaum O. The tumor microenvironment in the post-PAGET era. Cancer letters. 2006; 242:1-10.

41. Aguirre-Ghiso JA. Models, mechanisms and clinical evidence for cancer dormancy. Nature reviews Cancer. 2007; 7:834-846.

42. Joyce JA and Pollard JW. Microenvironmental regulation of metastasis. Nature reviews Cancer. 2009; 9:239-252.

43. Witz IP. Yin-yang activities and vicious cycles in the tumor microenvironment. Cancer research. 2008; 68:9-13.
44. Maman S, Edry-Botzer L, Sagi-Assif O, Meshel T, Yuan W, Lu W and Witz IP. The metastatic microenvironment: lung-derived factors control the viability of neuroblastoma lung metastasis. International journal of cancer. 2013; 133:2296-2306.

45. Rondeau G, Abedinpour P, Desai P, Baron VT, Borgstrom $\mathrm{P}$ and Welsh J. Effects of different tissue microenvironments on gene expression in breast cancer cells. PloS one. 2014; 9:e101160.

46. Salvatore V, Teti G, Bolzani S, Focaroli S, Durante S, Mazzotti MC and Falconi M. Simulating tumor microenvironment: changes in protein expression in an in vitro co-culture system. Cancer cell international. 2014; $14: 40$.

47. Herman JG, Jen J, Merlo A and Baylin SB. Hypermethylation-associated inactivation indicates a tumor suppressor role for p15INK4B. Cancer research. 1996; 56:722-727.

48. Merlo A, Herman JG, Mao L, Lee DJ, Gabrielson E, Burger PC, Baylin SB and Sidransky D. 5' CpG island methylation is associated with transcriptional silencing of the tumour suppressor p16/CDKN2/MTS1 in human cancers. Nature medicine. 1995; 1:686-692.

49. Teitz T, Wei T, Valentine MB, Vanin EF, Grenet J, Valentine VA, Behm FG, Look AT, Lahti JM and Kidd VJ. Caspase 8 is deleted or silenced preferentially in childhood neuroblastomas with amplification of MYCN. Nature medicine. 2000; 6:529-535.

50. van Hoesel AQ, Sato Y, Elashoff DA, Turner RR, Giuliano AE, Shamonki JM, Kuppen PJ, van de Velde CJ and Hoon DS. Assessment of DNA methylation status in early stages of breast cancer development. Br J Cancer. 2013; 108:2033-2038.

51. Geminder H, Sagi-Assif O, Goldberg L, Meshel T, Rechavi G, Witz IP and Ben-Baruch A. A possible role for CXCR4 and its ligand, the $\mathrm{CXC}$ chemokine stromal cell-derived factor-1, in the development of bone marrow metastases in neuroblastoma. Journal of immunology. 2001; 167:4747-4757.

52. Klein A, Schwartz H, Sagi-Assif O, Meshel T, Izraely S, Ben Menachem S, Bengaiev R, Ben-Shmuel A, Nahmias C, Couraud PO, Witz IP and Erez N. Astrocytes facilitate melanoma brain metastasis via secretion of IL-23. The Journal of pathology. 2015; 236:116-127. 